Supporting Information

\title{
Flocculation Efficiency and Spatial Distribution of Water in Oil Sands Tailings Flocculated with a Partially Hydrophobic Graft Copolymer
}

\author{
Gayathri Kalyanaraman ${ }^{\mathrm{a}}$, Zahra Rostami Najafabadi ${ }^{\mathrm{b}}$, João Soares ${ }^{\mathrm{b}}$, Milana Trifkovic ${ }^{\mathrm{a} *}$ \\ ${ }^{a}$ Department of Chemical and Petroleum Engineering, Schulich School of Engineering, University of \\ Calgary, 622 Collegiate P1 NW, Calgary, Alberta, T2N 4V8, Canada \\ b Department of Chemical and Materials Engineering, Donadeo Innovation Centre for \\ Engineering, University of Alberta, 9211-116 Street NW, Edmonton, Alberta, T6G 1H9, Canada \\ *Email: mtrifkov@ucalgary.ca
}

\section{List of Authors and Affiliations}

1. Milana Trifkovic ${ }^{\text {* }}$ (mtrifkov@ucalgary.ca)

Associate Professor

University of Calgary,

Department of Chemical and Petroleum Engineering,

Calgary, AB, Canada

\section{João Soares ${ }^{\text {b }}$}

Professor

University of Alberta,

Department of Chemical and Materials Engineering,

Edmonton, AB, Canada

\author{
3. Gayathri Kalyanaraman ${ }^{a}$ \\ Graduate Student \\ University of Calgary, \\ Department of Chemical and Petroleum Engineering, \\ Calgary, AB, Canada
4. Zahra Raostami Najafabadi ${ }^{b}$
Graduate Student
University of Alberta,
Department of Chemical and Materials Engineering,
Edmonton, AB, Canada


Number of Figures: 6

Number of Tables: 3

Pseudocode: 1

\section{Table of Contents}

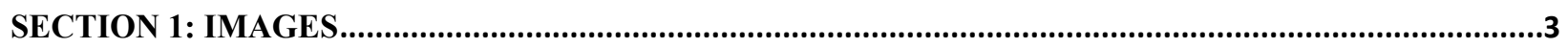

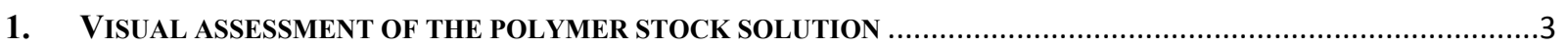

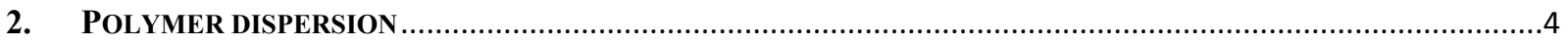

3. STRESS RELAXATION RHEOLOGY OF THE POLYMER FLOCCULANTS ….............................................

4. INITIAL EXPERIMENTS FOR DETERMINING THE OPTIMAL POLYMER DOSAGE:.....................................6

4.1 Visual Inspection of Mature Fine Tailings-MFT flocculated with 3 grafting densities of EPDM-gHPMA at various polymer concentrations. .......................................................................................6

4.2 Rheology and microstructure correlation for MFT flocculated with polymers at different

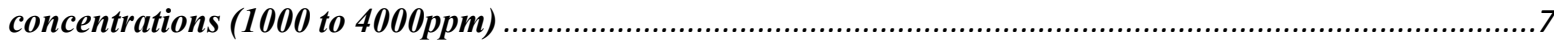

5. STUDY WITH OPTIMIZED FLOCCULANT DOSAGE (2000PPM) .......................................................... 5.1 Visual Inspection of Mature Fine Tailings-MFT flocculated with 3 grafting densities of EPDM-gHPMA 8

$5.2 \quad 3 D$ confocal imaging of the sediments and their compaction over aging .....................................

$5.3 \quad$ Rheology of 120 days aged sediment using Vane geometry .......................................................10

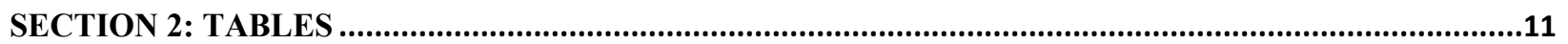

A. IMAGE PROCESSING-DATA QUANTIFICATION AT OPTIMIZED POLYMER DOSAGE (2000 PPM) .................11

B. DEAN STARK ANALYSIS-MASS BALANCE AT OPTIMIZED POLYMER DOSAGE (2000 PPM) .....................11

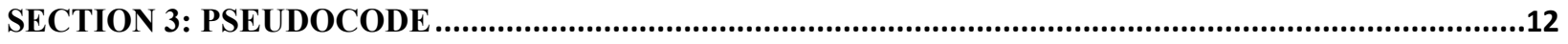

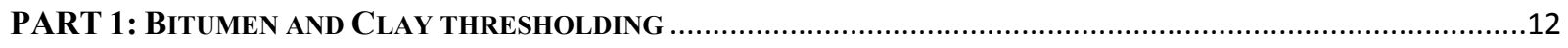

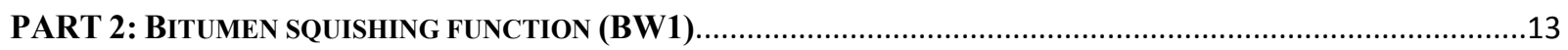




\section{Section 1: Images}

1. Visual assessment of the polymer stock solution

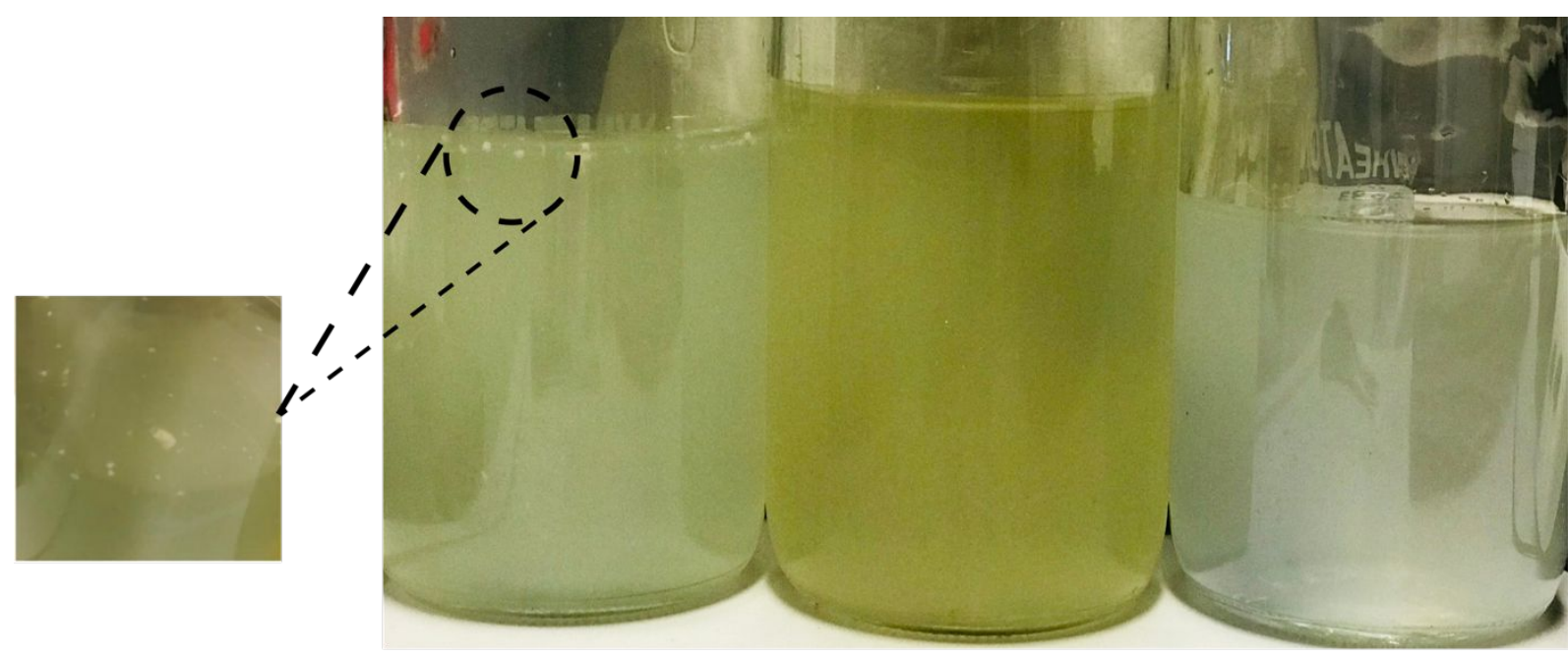

\section{EPDM-g-HPMA 30 EPDM-g-HPMA 40 EPDM-g-HPMA 50}

Figure S 1. Polymer Stock Solution, with EPDM-g-HPMA 30 having the highest concentration $(0.0105 \mathrm{~g} / \mathrm{ml})$ and showing the precipitation. The concentration of EPDM-g-HPMA 40 and 50 are $0.0089 \mathrm{~g} / \mathrm{ml}$ and $0.00306 \mathrm{~g} / \mathrm{ml}$, respectively. 


\section{Polymer dispersion}
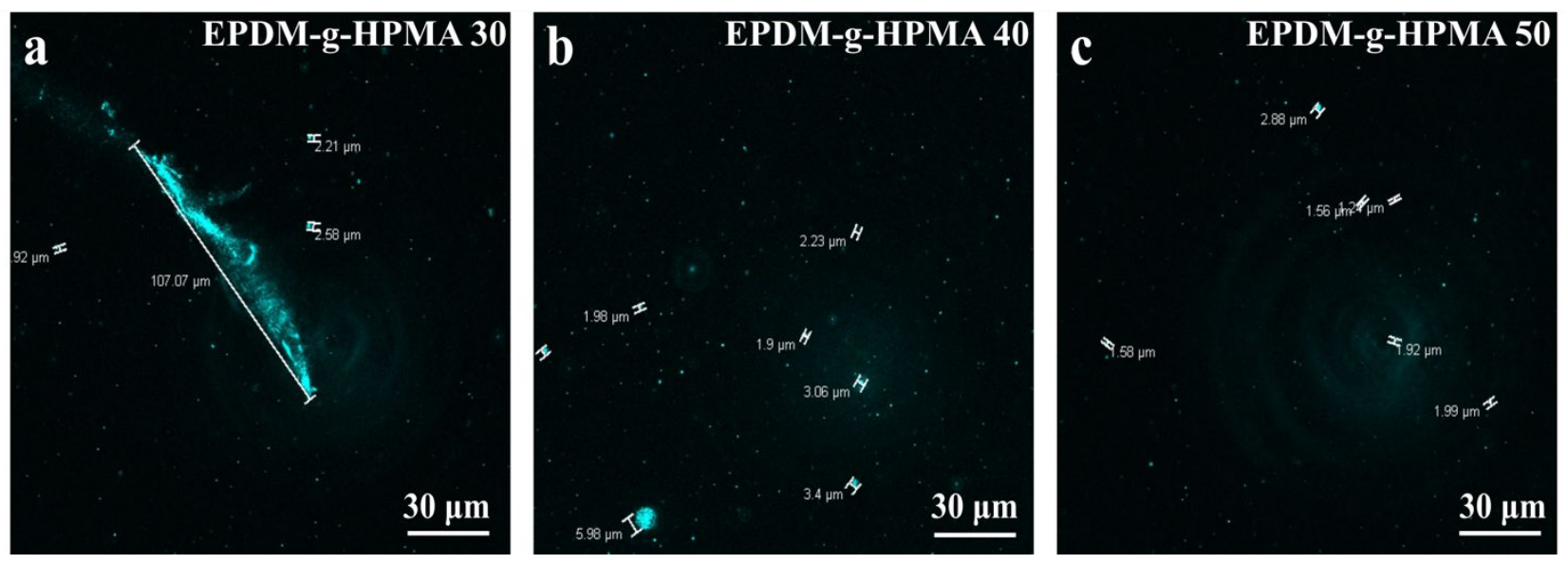

Figure S 2. 2D image of flocculant dispersion indicating the polymer chain aggregates and their sizing in microns. The images were acquired using Laser Scanning Confocal Microscopy (LSCM) using the methodology outlined in section 2.3.1 of our manuscript. The average size was computed by mathematically averaging the size of each aggregate from the imaging data. 
3. Stress relaxation rheology of the polymer flocculants

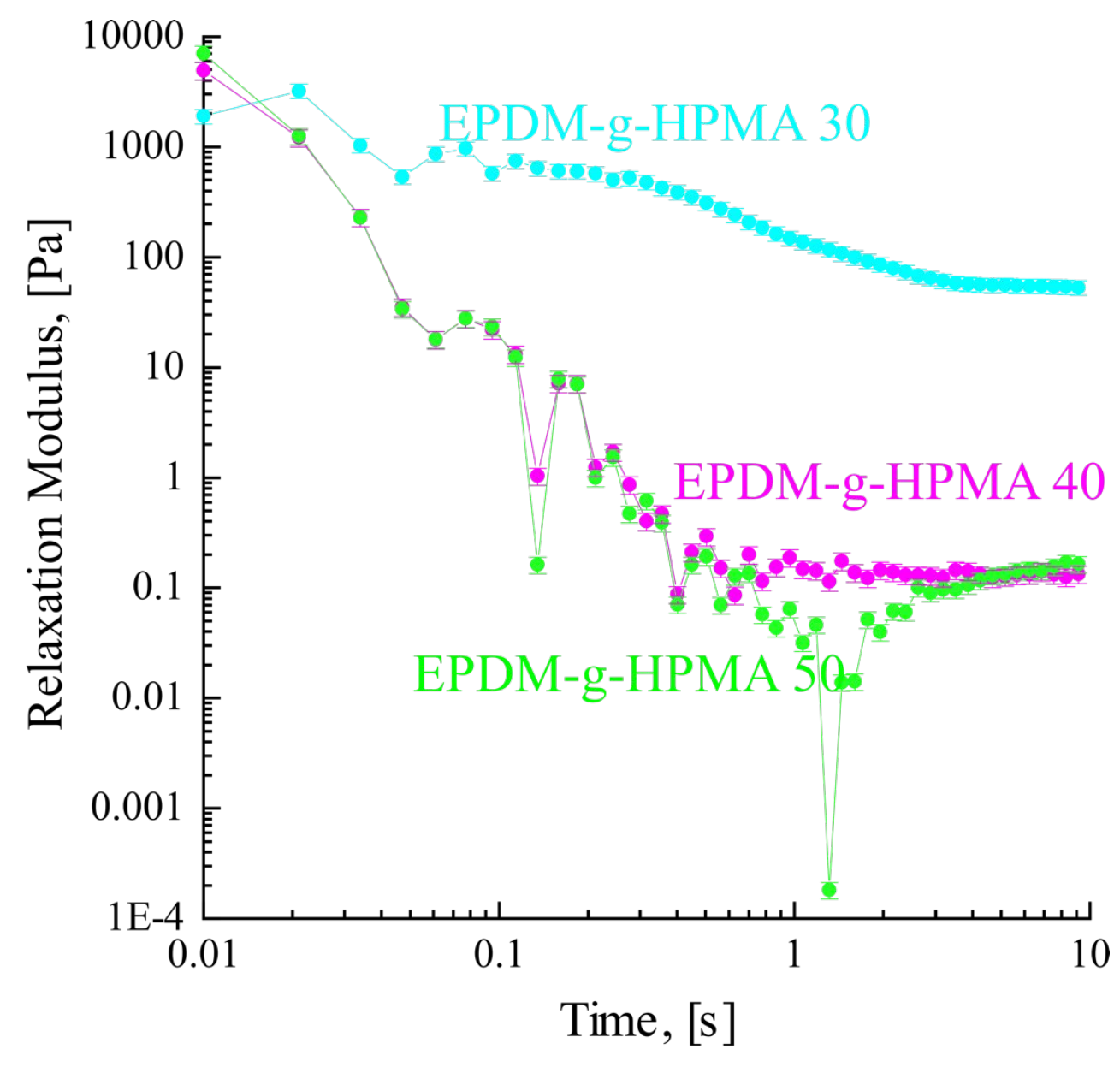

Figure S 3.Stress Relaxation Rheology on the flocculants (raw polymers). The poor dispersion of the EPDM-g-PMA 30 makes it hard to relax in comparison to the well-relaxed polymers (EPDMg-PMA 40 and 50) 


\section{Initial Experiments for determining the optimal polymer dosage:}

\subsection{Visual Inspection of Mature Fine Tailings-MFT flocculated with 3 grafting densities of EPDM-g-HPMA at various polymer concentrations.}

EPDM-g-HPMA 30

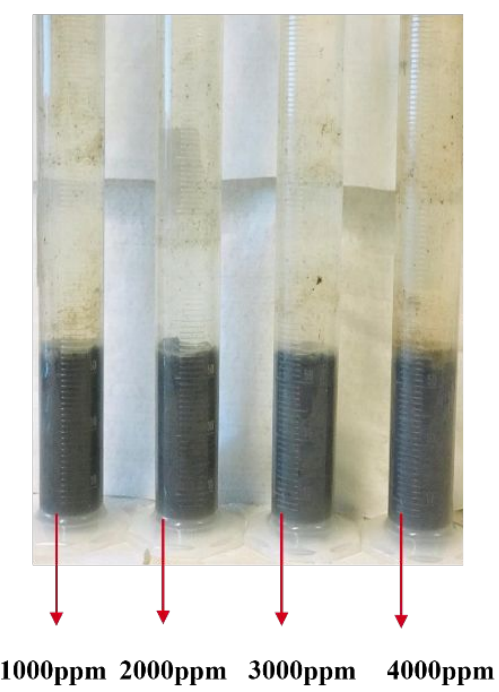

EPDM-g-HPMA 40

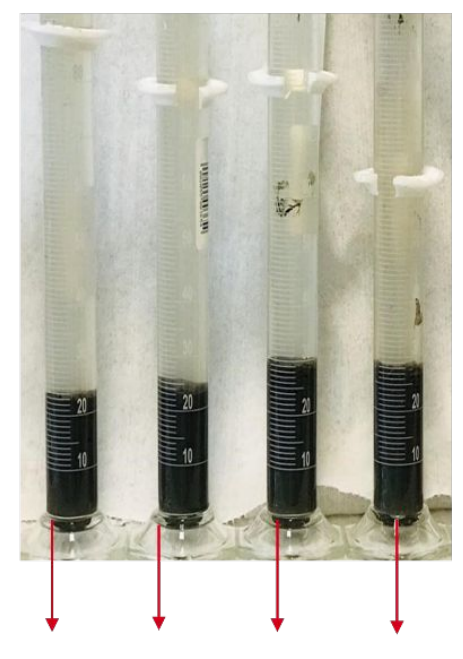

EPDM-g-HPMA 50

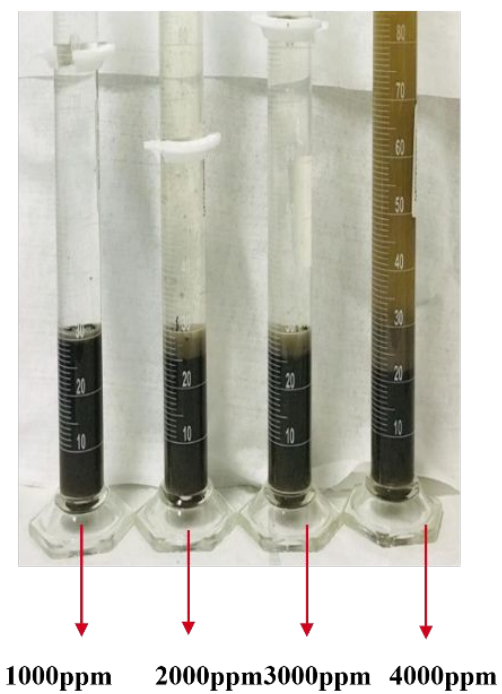

Figure S 4. Visual inspection of MFT flocculated with 1000ppm 2000 ppm, 3000 ppm and 4000 ppm of: EPDM-g-HPMA-30, 40, 50. Pictures were taken 24 hours after flocculation. The image indicates that, with an increase in polymer concentration, the turbidity of the solution increases. This led us to choose the polymer with a lower concentration. 


\subsection{Rheology and microstructure correlation for MFT flocculated with polymers at different concentrations (1000 to $4000 \mathrm{ppm})$}

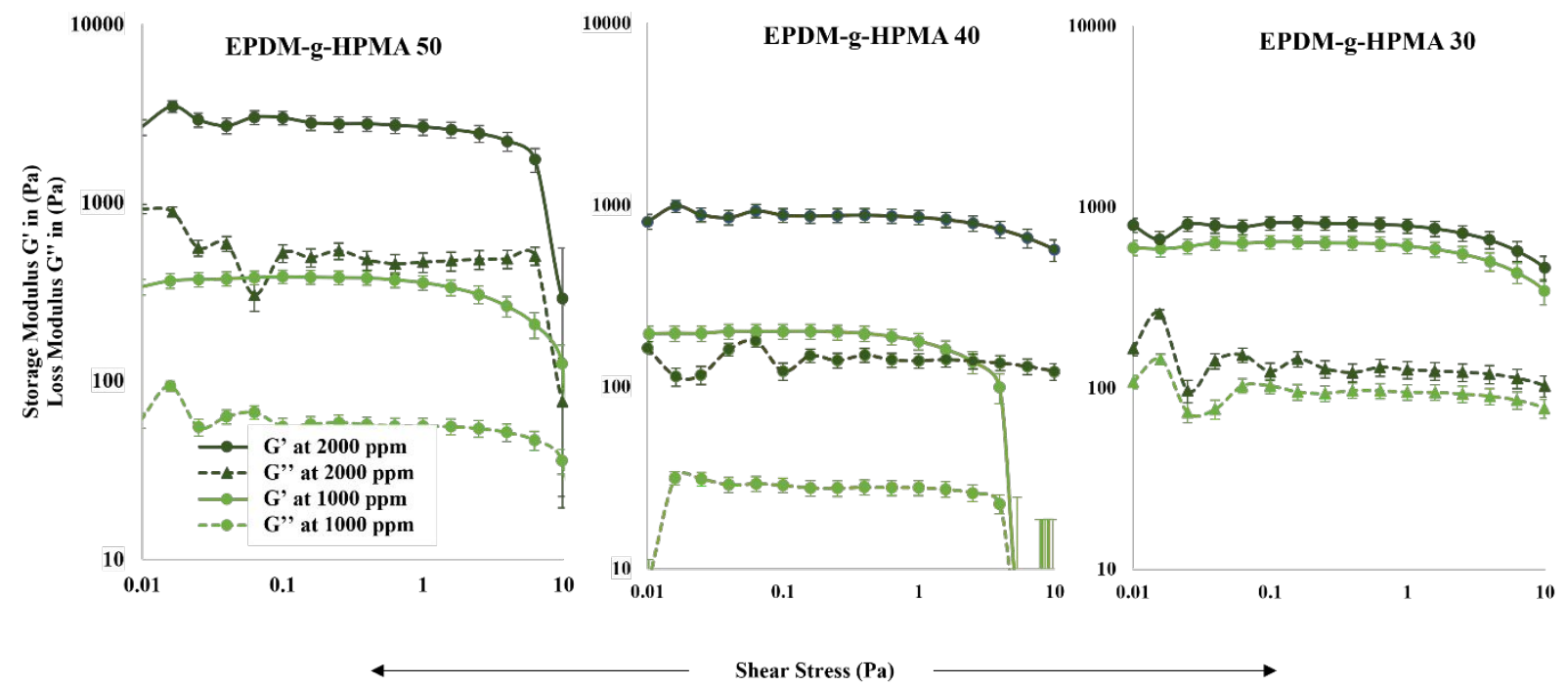

Figure S 5. Rheology indicating the storage and loss modulus of MFT sediments flocculated with 1000ppm, and 2000 ppm, of EPDM-g-HPMA-30, 40, 50. Rheology was performed seven days after flocculation. The results indicate that the strength of the sediment increases with an increase in grafting density, increase in polymer concentration, and the solids compaction follows the same order. 
5. Study with optimized flocculant dosage (2000ppm)

5.1 Visual Inspection of Mature Fine Tailings-MFT flocculated with 3 grafting densities of EPDM-g-HPMA

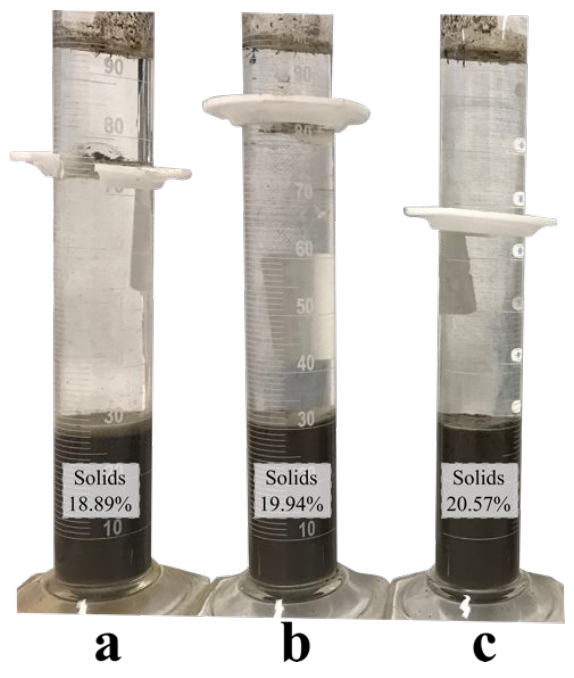

Figure S 6. Visual inspection of MFT flocculated with 2000 ppm of: a) EPDM-g-HPMA-30, b) EPDM-g-HPMA-40, c) EPDM-g-HPMA-50. Pictures were taken 24 hours after flocculation. The solids content were obtained by drying the flocculated sediments overnight in a vacuum oven and by using equation (1) mentioned in the manuscript. 


\subsection{D confocal imaging of the sediments and their compaction over aging}

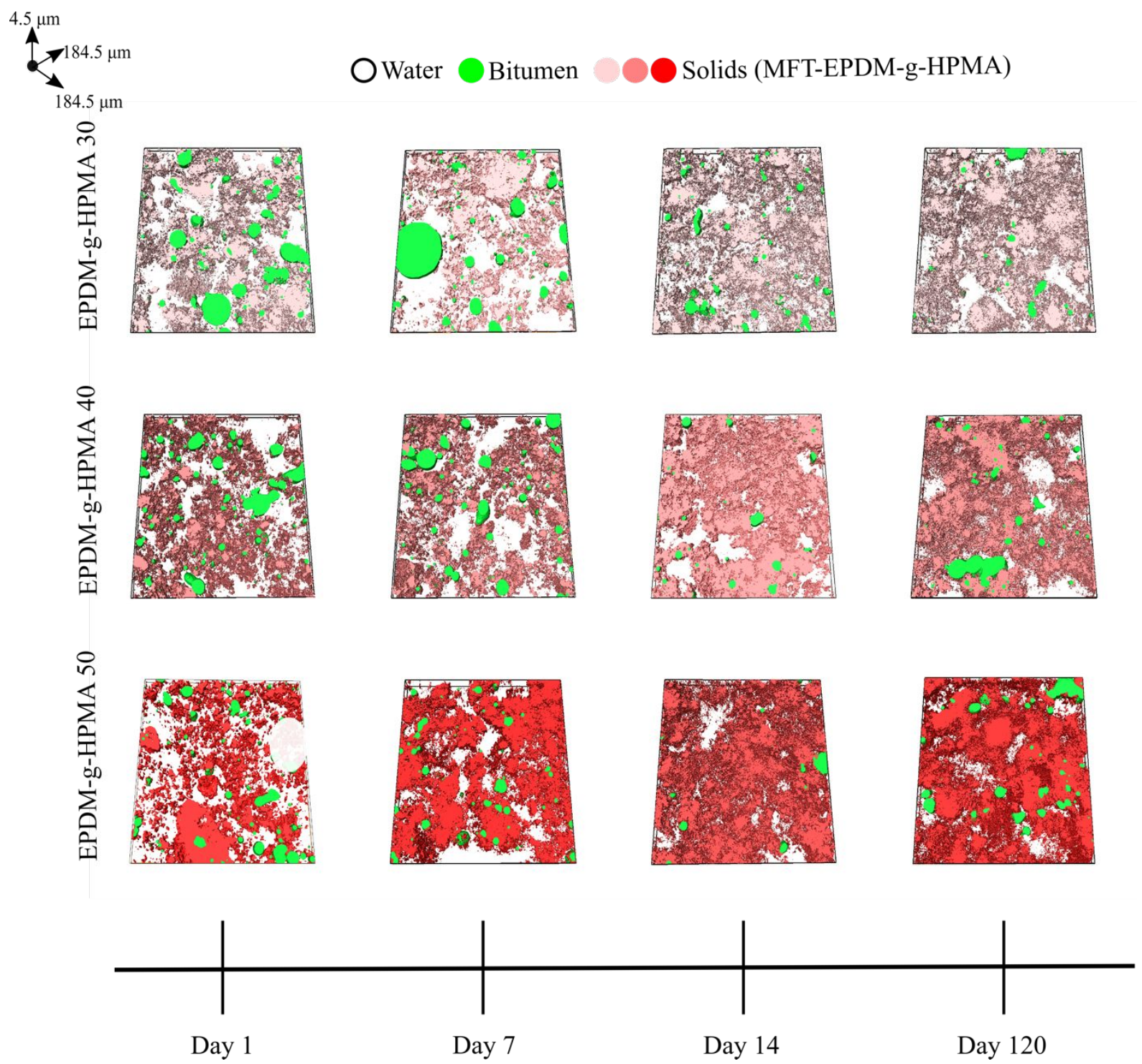

Figure S 7: 3D structure of the flocculated MFT sediment each at 2000ppm dosage of polymer flocculant showing the localization of clay (red), bitumen (green) and water (white) components within the porous sediment network. The dark, medium, and light red colour codes correspond to the polymers EPDM-g-PMA 50, 40 and 30. More compact structure evolution with aging is observed for all three cases. 


\subsection{Rheology of 120 days aged sediment using Vane geometry}

The rheology studies of 120 days aged sediment were performed using vane geometry in addition to the concentric cylinder measuring system. The vane geometry results were $\sim 2 \mathrm{k} \mathrm{Pa}$ less than the ones obtained from the concentric cylinder data.

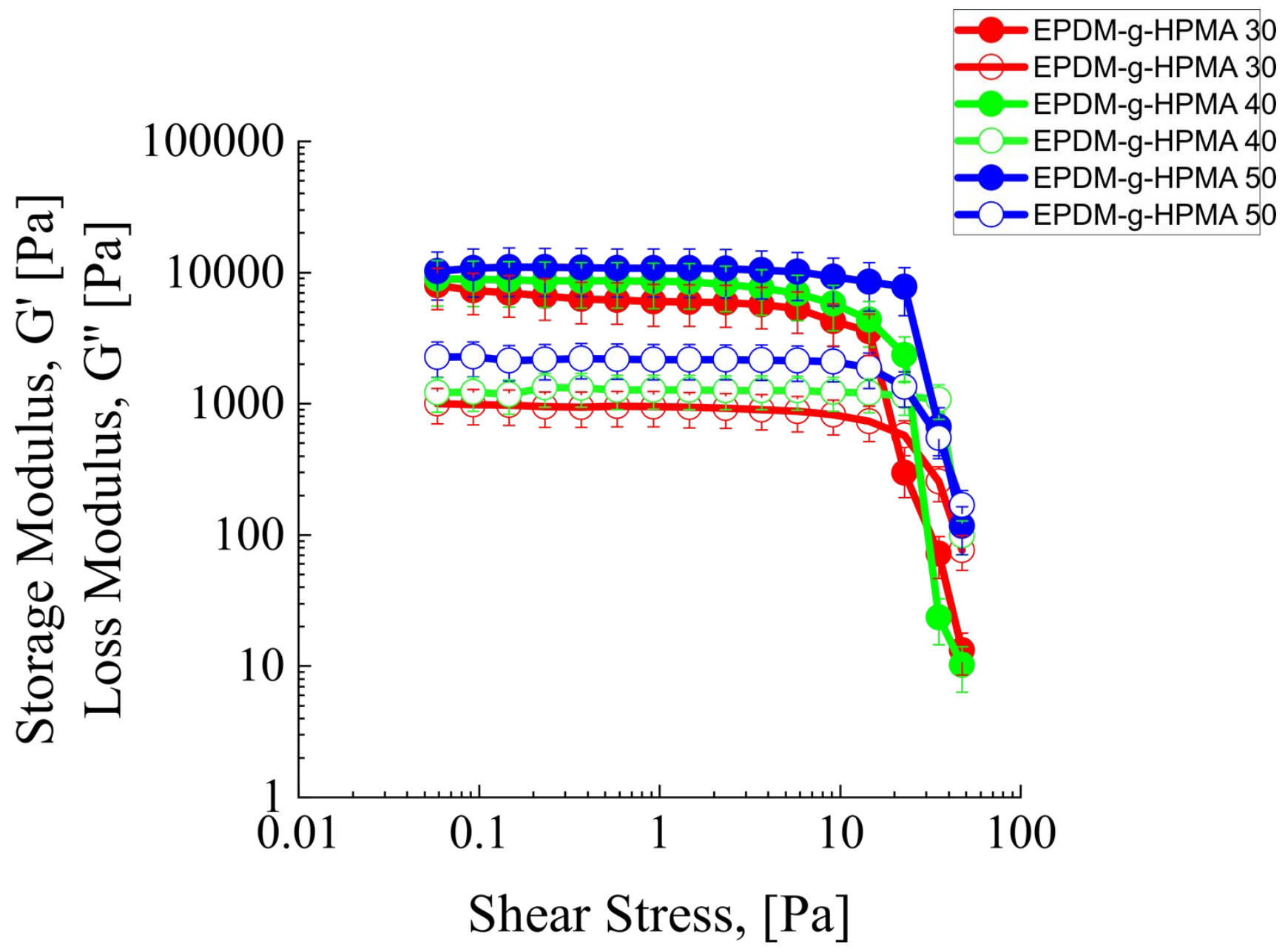

Figure S 8. Rheological data for the 120 days aged sample obtained using vane geometry. 


\section{Section 2: Tables}

\section{a. Image processing-data quantification at optimized polymer dosage $(2000 \mathrm{ppm})$}

\begin{tabular}{|c|c|c|c|c|c|c|c|c|c|c|c|c|}
\hline Variables: & DAY-0 & & & DAY-7 & & & DAY-14 & & & DAY-120 & & \\
\hline Solids vol \% & 23.73 & 24.56 & 27.56 & 24.99 & 26.13 & 29.36 & 26.22 & 30.73 & 33.68 & 32.33 & 36.03 & 41.18 \\
\hline Bitumen vol \% & 6.31 & 7.46 & 5.08 & 5.12 & 6.67 & 4.73 & 4.01 & 2.37 & 1.63 & 2.09 & 3.58 & 3.35 \\
\hline Water vol \% & 69.96 & 67.98 & 67.36 & 69.89 & 67.2 & 65.91 & 69.77 & 66.9 & 64.69 & 65.58 & 60.39 & 55.47 \\
\hline Fractal Dimension & 2.5680 & 2.6003 & 2.6226 & 2.5838 & 2.6651 & 2.7248 & 2.6729 & 2.7184 & 2.7683 & 2.7253 & 2.7535 & 2.7814 \\
\hline
\end{tabular}

Table S 1.Quantified data of the structural parameters obtained from the image post-processing step.

\section{b. Dean Stark analysis-mass balance at optimized polymer dosage (2000 ppm)}

\begin{tabular}{|c|c|c|c|c|c|c|c|c|c|c|c|c|c|}
\hline & & EPDM - & -HPM & & & EPDM & -HPM & 40 & & EPDM & -HPM & & \\
\hline Water & 1.00 & 19.57 & 57.61 & 19.57 & 72.98 & 30.56 & 53.21 & 30.56 & 70.59 & 10.71 & 50.12 & 10.71 & 68.22 \\
\hline Bitumen & 1.05 & 1.91 & 5.61 & 1.82 & 6.77 & 2.85 & 4.97 & 2.71 & 6.28 & 0.87 & 4.08 & 0.83 & 5.28 \\
\hline Intra floc & & $\begin{array}{l}=72.98-65 \\
=7.40\end{array}$ & & & & $\begin{array}{l}=70.59-6 \\
=10.20\end{array}$ & & & & $\begin{array}{l}=68.22-5 \\
=12.75 \%\end{array}$ & & & \\
\hline
\end{tabular}

Table S 2.Data of Dean-Stark analysis obtained through mass balance calculations. 


\section{Section 3: Pseudocode}

The first part of the coding shows the bitumen and clay channel's thresholding and their respective binary images. The second part shows the coding function for squishing the bitumen that has been applied in part 1 . The thresholded binary images were used for constructing the 3D surface of the flocculated sediments.

\section{PART 1: Bitumen and Clay thresholding}

BEGIN

INPUT

READ

FOR each image in $\mathrm{J}$ less or equal to $\mathrm{z}$.

$\mathrm{F} 1(\mathrm{j}) \mathrm{i}$ - reads set of bitumen channel-1 images at $\mathrm{z}=\mathrm{i}$

$\mathrm{F} 2(\mathrm{~J})_{\mathrm{i}}$ - reads set of clay channel-2 images at $\mathrm{z}=1$

\section{BITUMEN}

READ bitumen channel-1 images at height " $z$ " from F1(j)

APPLY average filter to enhance bitumen channel-1 at height $\mathrm{z}$

BINEARIZE enhanced image using Otsu's method

FILL voids and spaces

REMOVE small and noisy objects

OUTPUT. BW1 binary images of bitumen channel-1

CLAY

READ clay channel-2 images at height " $z$ " from F1(J)

ENHANCE the clay channel's image contrast at height " $z$ "

APPLY average filter to enhance clay channel-2 images at $\mathrm{z}=\mathrm{i}$

DETERMINE VOIDS

OPEN image

ERODE image

RECONSTRUCT image

CLOSE image

DILATE image

RECONSTRUCT the dilated image

BINEARIZE using Otsu's method

CLOSE image

DETERMINE EDGES using Sobel operator

FILL the voids and interior spaces

SMOOTH objects

RECONSTRUCT objects using the above obtained information

OUTPUT.BW2 binary images of clay channel-2

END FOR 
CALL the function bitumen squishing (BW1)

OBTAIN squished bitumen (channel-1)

REMOVE the overlaid bitumen from the binarized clay channel

SAVE respective channel's binary images at each " $z$ " in the format tiff END

\section{PART 2: Bitumen squishing function (BW1) \\ READ BW1}

REMOVE small objects

FIND connected components

MEASURE the properties of the found components

SET SIZE and smillarity PARAMETER

FOR each connected component

FIND centre

FOR each layer of connected element

IF layer $==$ reference layer

REMOVE current layer

OTHERWISE

REPLACE with current layer

SAVE new reference layer in auxillary matrix

END IF

END FOR

IF the object is in contact with the top or bottom layer

DO NOT MOVE the connected component

OTHERWISE

MOVE object to calculated centre

END IF

SAVE location, size and morphology of the squished connected bitumen at OUT

RETURN (OUT)

END FUNCTION 